\title{
Public and Private Provision of Health Care*
}

\author{
Pedro Pita Barros \\ Universidade Nova de Lisboa \\ CEPR (London)
}

\author{
Xavier Martinez-Giralt \\ Universitat Autònoma de Barcelona
}

March 2000

\begin{abstract}
One of the mechanisms that is implemented in the cost containment wave in the health care sectors in western countries is the definition, by the third-party payer, of a set of preferred providers. The insured patients have different access rules to such providers when ill. The rules specify the co-payments and the indemnity the patient obtains if patronizing an out-of-plan care provider. We propose to study the competitive process among providers in terms of both prices and qualities. Competition is influenced among other factors by the status of providers as in-plan or out-of-plan care providers. Also, we face a moral hazard of provider choice related to the trade-off between freedom to choose and the need to hold down costs.

Our main findings are that we can define a reimbursement scheme when decisions on prices and qualities are taken simultaneously (that we relate to primary health care sectors) such that the first-best allocation is achieved. In contrast, some type of regulation is needed to achieve the optimal solution when decisions are sequential (specialized health care sector). We also derive some normative conclusions on the way price controls should be implemented in some European Union Member States.
\end{abstract}

JEL Numbers: I11, I18

Keywords: Mixed oligopoly, health care.

CORRESPONDENCE ADDRESSES:

Pedro Pita Barros

Xavier Martinez-Giralt

Universidade Nova de Lisboa

Departamento de Economia

Travessa Estêvão Pinto

CODE and

P-1099-032 Lisboa, Portugal

Fax: 351-21-388 6073

Departament d'Economia i d'Història Econòmica

Universitat Autònoma de Barcelona

08193 Bellaterra, Spain

Email: ppbarros@fe.unl.pt

Fax: 34-93-581 2012

xavier.martinez.giralt@uab.es

${ }^{*}$ We benefited from useful comments and suggestions of Matilde Machado, Inés Macho-Stadler, Pau Olivella, José M. Usategui and two anonymous referees. We acknowledge the financial support of SGR98-0062 and HP97-0062 (Xavier Martinez-Giralt) and AILE E58/98 (Pedro Pita Barros). The usual disclaimer applies. 


\section{Introduction}

A common characteristic of the Welfare State in the OECD countries is the desire (need) to reform the public health care system. This arises from the increasing difficulties in financing the system. Also, within the European Union, the Maastricht criteria (particularly on budget deficits) to participate in the EMU area has created an additional pressure to lower public expenditures. This pressure is calling into question the so-called universal system of public health care. New organizational forms to improve efficiency are being tested, such as the private management of public hospitals that is being implemented in Spain and Portugal. ${ }^{1}$

Due to the elements of moral hazard involved in the health care insurance contracts, third-party payers are implementing mechanisms to control for expenses. One of these mechanisms is the definition of a set of providers to which the insured patients have access to obtain treatment when ill. Associated with this goes the definition of the indemnity the patient obtains should (s)he address a provider outside that set.

Surprisingly enough, there is very little literature on the process of selecting providers and on competition among providers when different reimbursement rules apply, according to the provider chosen by the patient. Our present paper specifically addresses this last issue. ${ }^{2}$

The results and their implications are of interest to insurers (private or public) whenever they set special agreements with a subset of providers, as is the case in some managed care experiments, and to National Health Services that use private providers (they may also define preferred sets of providers) and/or have own provision, which competes with private providers.

Our paper deals with the competitive effects on providers of different reimbursement rules. They translate into being included or excluded in the list of selected providers by an insurer, which, in turn, will have an impact on their decisions regarding quality and price. Also, we assume that all our providers are always active in the market. Generally, patients have to bear part of the cost of the treatment provided by an in-plan care provider. If, instead (s)he visits an out-of-plan care provider, (s)he pays the full price and obtains the indemnity from the insurer specified in the insurance contract.

We will consider three basic alternatives for the indemnity associated with the out-of-plan provider. The first one simply does not provide coverage for choices outside the "preferred provider" set. This captures a pure public system of health provision, such as the Spanish one, where a patient visiting a private provider (instead of a public one) has to bear the full cost of the treatment. The second

\footnotetext{
${ }^{1}$ Other examples of experiments aimed at cost containment can be found in Le Grand and Mossialos (1999).

${ }^{2}$ A companion paper Barros and Martinez-Giralt (2000) addresses the former one.
} 
alternative defines an indemnity equal to what the patient would have obtained should (s)he visited a preferred provider. This alternative tries to capture the idea of indemnity based on a reference price. This captures some features of the French system. Also, it captures some important features of the pharmaceutical sector. Finally, the third alternative is equivalent to the scenario where both providers have been selected by the insurer. This captures some features of the German system where together with the public providers, there is a fringe of private providers regulated through bilateral agreements.

The type of questions we address refer to the characteristics of the market allocations according to the type of insurance contract offered by the insurer and to different assumptions about the timing of the decisions on prices and qualities taken by the providers.

We will then turn our attention to a market where the preferred provider acts as a leader. First, we will consider an agency regulating both price and quality of the public provider acting as a Stackelberg leader and the private provider as a follower. This should capture the present type of regulation in health care markets such as in Spain. Finally, we envisage a possible regulation of the health sector in the form of a three-stage game where the regulator sets the level of quality to maximize welfare, then the private provider decides its quality level and finally both providers compete in prices. We address the issue of whether public leadership, coupled with out-of-plan rules, are sufficient instruments to achieve the first-best allocation.

Accordingly, Section 2 presents the basic framework. Section 3 reports the main features associated with price competition. Section 4 sets the social optimum allocation, which will serve as a benchmark. Section 5 reports the analysis of both quality and price decisions. Section 6 deals with leadership by a public provider. Finally, section 7 concludes the paper.

\section{The model}

There is very little literature linking health insurance and differentiation in the health care market. Ma and Burgess (1993) consider a model combining vertical and horizontal differentiation to study the characteristics of equilibrium allocations under sequential decisions first on quality then on prices (locations are given). The authors show that sequentiality of decisions creates a strategic effect that distorts decisions relative to socially optimal decisions. Exploring the efficiency properties of two regulatory policies, Ma and Burgess conclude that a two-part tariff yields better results than does a simple regulation of prices. A similar strategic effect of sequential moves is also present in some of the variants of our model. We depart from Ma and Burgess on the set of instruments addressed: we look at the reimbursement rules of the health plan. 
In a more recent paper, Che and Gale (1997) propose a similar model where a principal decides between two types of competition. Ex-ante competition approximates the idea of managed competition in that providers propose quality-price pairs to the principal. Then, the principal decides which providers will be active in the market. Ex-post competition simply consists of the providers proposing those pairs directly to consumers. The authors conclude with a preference for the ex-ante competition because it allows for the extraction of all the surplus from the providers, although it involves too many providers offering too much quality with respect to the optimal values.

Finally, Wolinsky (1997) proposes a scenario of managed competition where providers compete in quality vs. a situation of a segmented market where there is a regulated monopolist in each submarket. The aim of the paper is to find the conditions under which every situation is superior to the alternative one. ${ }^{3}$

In the definition of the model, we must draw attention to some stylized facts. The first one is horizontal product differentiation of providers. The second one is the importance of quality. Isaacs (1996) points out that the choice of provider by the patient is the most difficult decision for the patient in informational terms. The location of the hospital is usually the crucial element for most patients. The reason being that although quality is regarded as important, different indices of quality convey different rankings of providers. Also, it is not always easy to understand the meaning of some of these indicators. In contrast, the pricing policy and the location are relatively easy to compare.

The model we propose combines elements of vertical differentiation (quality) and horizontal differentiation in a set up à la Hotelling. Qualities are selected by providers. These are observable but not contractible. They are the source of moral hazard. Patients obtain utility from their treatment. This utility is increasing in the level of quality supplied by the provider and on the indemnity; it is decreasing on the price and on the transport cost. Transport cost may be understood either in terms of the cost to move from the patient's location to the hospital or in utility terms as the loss in utility when the patient does not have access to his most preferred hospital. Insurers have already defined a set of preferred providers to supply health services to their insured population. The insurance contract specifies a co-payment that may vary according to the provider selected and the indemnity should the patient address a provider outside of the health plan. Health insurance is assumed to be compulsory.

There are two providers in the market for provision of health care. Consumers have preferences over providers. To describe these preferences, we assume providers to be located at the endpoints of the segment $[0,1]$.

\footnotetext{
${ }^{3}$ See also the introduction to the JEMS second special issue on the industrial organization of health care by Ma and McGuire (1997).
} 
Consumers benefit from a health insurance system (public or private), heretofore the insurer, which provides an indemnity in the case of illness. When ill, consumers demand one unit of health care. In each period, there are consumers in every point of the segment $[0,1]$ that need care. That is, consumers are uniformly distributed in terms of their preferences for providers along the line $[0,1]$, with density one.

The insurer has an arrangement with provider $A$ (located at zero), which implies that individuals pay only $c \%$ of price in case of choosing this provider. The remaining $(1-c) \%$ of total health care cost is paid by the insurer. We assume that $c>0$, that is, insurance contracts do not provide full insurance. If the consumer chooses the other provider, several options may have been specified in the insurance contract: (i) the consumer has to pay the full price (no insurance is given); (ii) the insurer pays the amount that it would pay were provider $A$ chosen (as long as the price of provider $B$ exceeds that amount, otherwise it pays the amount charged by provider $B$ ); and (iii) give the same co-insurance rate, meaning that no preferential treatment exists. This last case corresponds to a system where the insurer treats both providers equally, and in fact provides the same insurance level whichever provider is selected. There is no preferred set of providers. The three cases differ only in the amount of reimbursement given to consumers who choose provider $B .^{4}$

Providers set prices and qualities of the services provided freely, unless otherwise stated. Denote by $P_{i}$ the price and by $T_{i}$ the quality selected by provider $i, i=A, B$. The production cost of health care is normalized to zero. The utility of a consumer located at $x$ when choosing provider $i, V(i, x), i=A, B$ is given by

$$
V(A, x)=Y+T_{A}-P_{A} c-t x ; \quad V(B, x)=Y+T_{B}+I-t(1-x)-P_{B}
$$

where $Y$ is income, $t x$ is the cost of going to provider $A$, measured in monetary units (similarly, $t(1-x)$ is the transport cost of choosing provider $B)$, and $I$ is the indemnity received when selecting the out-of-plan care provider (provider $B$ is our model). It is required that $I<P_{B}$ for consistency with the less-than-full insurance assumption $(c>0)$. In the above-mentioned cases, we have:

- (i) $I=0$; This is denoted as "pure preferred" provider system (PP);

- (ii) $I=(1-c) P_{A}$; It is called "fixed co-payment" system (FC);

- (iii) $I=(1-c) P_{B}$; This is named "fixed reimbursement rate" system (FRR).

\footnotetext{
${ }^{4}$ As stated above, in this paper we are not dealing with providers' fees in the spirit of e.g. Glazer and McGuire (1993). We address this issue in our companion paper, instead.
} 
The three cases can be easily included in the slightly more general specification:

$$
I=\alpha(1-c) P_{A}+\beta P_{B}(1-c), \quad \alpha \in[0,1], \beta \in[0,1], \alpha+\beta \leq 1
$$

Case (i) implies ( $\alpha=0, \beta=0)$, case (ii) implies ( $\alpha=1, \beta=0)$, and case (iii) occurs for $(\alpha=$ $0, \beta=1)$. A consumer goes to provider $A$ if $V(A, x) \geq V(B, x)$. This allows us to define $x^{\prime}$ such that for $x \leq x^{\prime}$ consumers select provider $A$, while for $x>x^{\prime}$ provider $B$ is selected.

\section{Price Competition}

Take qualities as given and equal, $T_{i}=\bar{T}, i=A, B$. The demand each provider faces is determined by the location of the consumer indifferent between provider $A$ and provider $B$. The indifferent consumer is defined by

$$
x=\frac{1}{2}+\frac{\kappa P_{B}-\mu P_{A}}{2 t}
$$

where $\mu=c+(1-c) \alpha, \kappa=1-\beta(1-c), \mu \in[c, 1], \kappa \in[c, 1]$. Parameter values for the different cases are summarized in Table 1.

Table 1: Critical parameter values

\begin{tabular}{|l|l|ll|}
\hline & & $\kappa$ & $\mu$ \\
\hline FRR & $\alpha=0, \beta=1$ & $c$ & $c$ \\
FC & $\alpha=1, \beta=0$ & 1 & 1 \\
PP & $\alpha=0, \beta=0$ & 1 & $c$ \\
\hline
\end{tabular}

Demand directed to provider $A$ is simply $x$, while demand faced by provider $B$ is $1-x$. Under the assumption of zero production costs, profits of each provider are:

$$
B_{A}=x P_{A} ; \quad B_{B}=(1-x) P_{B}
$$

The system of first-order conditions for profit maximization on price decisions can be solved to get the equilibrium prices:

$$
P_{A}=t / \mu ; \quad P_{B}=t / \kappa
$$

The following characterization comes out directly:

$$
P_{A} \geq P_{B}, \frac{\partial P_{A}}{\partial \alpha}<0, \frac{\partial P_{A}}{\partial \beta}=0, \frac{\partial P_{B}}{\partial \alpha}=0, \frac{\partial P_{B}}{\partial \beta}>0 \frac{\partial P_{a}}{\partial c}<0, \frac{\partial P_{B}}{\partial c}<0, x=\frac{1}{2}
$$

The equilibrium prices were obtained under the assumption that the requirement $I<P_{B}$ holds. It is straightforward to show it to be implied, evaluated at equilibrium values, by $c>0$ (the no-full 
insurance assumption). Equality of equilibrium prices holds for the corner case of $\alpha+\beta=1$, which occurs for both fixed reimbursement and same co-insurance rate to consumers.

Define total payments of the health care system as $T C=P_{A} x+P_{B}(1-x)$, which yields, at equilibrium values, for the different cases, the amounts reported in Table 2. Some qualitative features emerge clearly. First, for a mixed health care provision market, it does, indeed, matter how reimbursement is made to the private provider. In particular, the way the insurer pays when consumers opt the out-of-plan care provider influences the degree of competition in the market.

Table 2: Equilibrium under different rules

\begin{tabular}{|ccccc|}
\hline \multicolumn{2}{|c}{ Reimbursement to } & & & \\
Provider $A$ & Provider $B$ & Prices & Profits & Total payments \\
\hline$(1-c) P_{A}$ & 0 & $P_{A}=t / c, P_{B}=t$ & $B_{A}=t / 2 c, B_{B}=t / 2$ & $(1+1 / c) t / 2$ \\
$(1-c) P_{A}$ & $(1-c) P_{A}$ & $P_{A}=P_{B}=t$ & $B_{A}=B_{B}=t / 2$ & $t$ \\
$(1-c) P_{A}$ & $(1-c) P_{B}$ & $P_{A}=P_{B}=t / c$ & $B_{A}=B_{B}=t / 2 c$ & $t / c$ \\
\hline
\end{tabular}

Setting $(\alpha=1, \beta=0)$ gives the same amount of reimbursement, defined by preferred provider, to whatever provider the consumer selects. This implies a lower payment to be made by the insurer than providing no insurance if an outside provider is chosen. The intuition behind the result is clear. Giving no insurance to outside providers softens competition to the preferred provider, which naturally induces it to carry higher prices. Reimbursement of some amount to the outsider provider creates tougher competition between providers, creating the conditions for lower prices. Note that in our stylized model, all effect is carried out in the price of the selected provider. This needs not to be true in more general settings. ${ }^{5}$

Another result obtained is that, according to the criterion of minimizing overall payments, excluding reimbursement to the outside provider's consumers is better than giving a co-insurance rate $c$, independent of the provider selected by the consumer. The reason is, again, the effect on the degree of competition, induced by giving some reimbursement to outside providers. On the other hand, giving the same co-insurance rate whichever provider is selected, softens price competition between providers, which results in a higher price level. The basic intuition is that making the amount reimbursed (not the rate of coverage) equal for both providers maintains competition at the margin. This basic mechanism will be present in the more general framework with quality choices, explored below.

\footnotetext{
${ }^{5}$ Extending the model to allow the possibility of the price being negotiated with the insurer does not eliminate this effect.
} 


\section{Social welfare}

We define welfare as the sum of consumer surplus and provider surplus, i.e.

$$
\begin{aligned}
W & =\int_{0}^{x}\left(Y+T_{A}-t \chi\right) d \chi+\int_{x}^{1}\left(Y+T_{B}-t(1-\chi)\right) d \chi-\phi\left(T_{A}\right)-\phi\left(T_{B}\right) \\
& =Y+T_{A} x+T_{B}(1-x)+t\left(x-x^{2}-\frac{1}{2}\right)-\phi\left(T_{A}\right)-\phi\left(T_{B}\right)
\end{aligned}
$$

where $\phi\left(T_{i}\right)$, with $\phi^{\prime}>0, \phi^{\prime \prime}>0$, is the cost function of quality level $T_{i}$ of provider $i$.

Implicit in this setting is the assumption that quality is a "public good" at each hospital. Although this assumption is probably applicable to many of the elements of quality our paper deals with, it also raises the possibility that only one provider might be optimal. ${ }^{6}$ We can advance two arguments to cope with this issue. On the one hand, our main interest here is to analyze the role of different reimbursement schemes on the competitive process among providers. On the other hand, if we are to understand the optimality of a single provider as just closing down one hospital, say B, and compare the welfare level achieved with provider $A$ located at zero versus our two providers, it is easy to check (assuming $\phi(T)=\theta T^{2} / 2$ ) that at the optimal value, two providers yield a higher level of welfare. This would not be the case if the planner is allowed not only to close down one provider but to choose the location for the remaining one as well. ${ }^{7}$

Now we analyze the optimal (first best) decision in terms of quality levels and demand distribution between the providers. That is, we look at the vector $\left(x, T_{A}, T_{B}\right)$ maximizing the welfare measure (2). The system of first-order conditions is given by,

$$
\begin{aligned}
T_{A}-T_{B}+t(1-2 x) & =0, \\
x-\phi^{\prime}\left(T_{A}\right) & =0, \\
(1-x)-\phi^{\prime}\left(T_{B}\right) & =0 .
\end{aligned}
$$

Solving for $x$, we obtain

$$
x=\frac{t+T_{A}-T_{B}}{2 t}
$$

Thus, the socially optimal quality choices are given by

$$
\frac{t+T_{i}-T_{j}}{2 t}=\phi^{\prime}\left(T_{i}\right), \quad i, j=A, B ; i \neq j .
$$

\footnotetext{
${ }^{6} \mathrm{We}$ acknowledge an anonymous referee for drawing our attention to this point.

${ }^{7}$ Of course, high-enough fixed costs will also make optimal the single-provider market structure. Still, under zero fixed costs, the point deserves some attention. Details on the argument can be found in 'http://ppbarros.fe.unl.pt/papers.html'.
} 
Given the symmetry of these conditions, in equilibrium it will be the case that $T_{A}=T_{B}=T^{s}$. Hence, the optimal value $T^{s}$ is determined by $\phi^{\prime}\left(T^{s}\right)=1 / 2$. It also results that $x^{s}=1 / 2$. This constitutes our welfare benchmark.

\section{Price and Quality Competition}

Having highlighted the main features associated with price competition and social optimum in the previous sections, we are now in a position to investigate the effect of different reimbursement rules on the choice of quality of providers, under two different timings for decisions. The natural interpretation of the simultaneity or sequentiality of decisions by a supplier of any good or service has to do with the distinction of (low-cost) short-run decisions versus (high-cost) long-run decisions. In our framework, a provider simultaneously deciding both the price and quality levels illustrates well a situation where the decision on quality is as easily reversible as the one regarding the price. This makes sense for primary care services (first-level providers) where investments in facilities are relatively small. In contrast, a sequential decision better illustrates the situation of specialized care services (second-level providers) where investment decisions in e.g. surgery rooms or high-tech instruments, are necessarily long-term decisions. Therefore, both simultaneous and sequential decisions on prices and qualities have some appeal.

Demands are again defined by the indifferent consumer:

$$
x=\frac{1}{2}+\frac{T_{A}-T_{B}}{2 t}+\frac{\kappa P_{B}-\mu P_{A}}{2 t},
$$

where $\mu$ and $\kappa$ carry the same expressions defined above. Profits of each firm are given by:

$$
B_{A}=P_{A} x-\phi\left(T_{A}\right) ; \quad B_{B}=P_{B}(1-x)-\phi\left(T_{B}\right) .
$$




\subsection{Simultaneous decisions}

Under simultaneous choices of prices and qualities by both providers, the equilibrium is given by the solution to the following set of first order conditions: ${ }^{8}$

$$
\begin{aligned}
\frac{\partial B_{A}}{\partial P_{A}} & =\frac{1}{2}+\frac{T_{A}-T_{B}}{2 t}+\frac{\kappa P_{B}-\mu P_{A}}{2 t}-\frac{\mu P_{A}}{2 t}=0, \\
\frac{\partial B_{A}}{\partial T_{A}} & =\frac{P_{A}}{2 t}-\phi^{\prime}\left(T_{A}\right)=0, \\
\frac{\partial B_{B}}{\partial P_{B}} & =\frac{1}{2}+\frac{T_{B}-T_{A}}{2 t}-\frac{\kappa P_{B}-\mu P_{A}}{2 t}-\frac{\kappa}{2 t} P_{B}=0, \\
\frac{\partial B_{B}}{\partial T_{B}} & =\frac{P_{B}}{2 t}-\phi^{\prime}\left(T_{B}\right)=0 .
\end{aligned}
$$

We solve first for equilibrium prices, conditional on qualities, and then for qualities. It is straightforward to show that:

$$
P_{A}=\frac{1}{\mu}\left(t+\frac{T_{A}-T_{B}}{3}\right) ; \quad P_{B}=\frac{1}{\kappa}\left(t+\frac{T_{B}-T_{A}}{3}\right) .
$$

Then, the quality decisions are characterized by:

$$
\begin{aligned}
& \left(t+\frac{T_{B}-T_{A}}{3}\right) \frac{1}{2 t \kappa}=\phi^{\prime}\left(T_{B}\right), \\
& \left(t+\frac{T_{A}-T_{B}}{3}\right) \frac{1}{2 t \mu}=\phi^{\prime}\left(T_{A}\right) .
\end{aligned}
$$

Each of these conditions defines a best-response function in the space $\left(T_{A}, T_{B}\right)$. These best-response functions are downward sloping, as qualities are strategic substitutes. Figure 1 illustrates the three equilibria.

For the case of fixed co-payment $(\alpha=1, \beta=0)$, it is the case that $T_{A}=T_{B}$, from which it follows that $P_{A}=P_{B}=t$. By comparative statics on first order conditions it is the case that

$$
\frac{\partial T_{A}}{\partial \alpha}<0, \frac{\partial T_{B}}{\partial \alpha}>0 ; \frac{\partial T_{A}}{\partial \beta}<0 ; \frac{\partial T_{B}}{\partial \beta}>0
$$

Consider now the scenario in which consumers pay the same proportion of the expenses (fixed reimbursement rate) they generate regardless of the provider at which they are attended. Take $(\beta=1, \alpha=$ 0 ), which implies that $\mu=\kappa=c$. The equilibrium qualities are defined by the solution to equations (8) and (9). From the symmetry of these two conditions, it must be the case that $T_{A}=T_{B}=T^{*}$ (and equilibrium prices are also equal). Hence, the solution to the optimal quality choice problem

\footnotetext{
${ }^{8}$ Second order conditions require $4 t \mu \phi^{\prime \prime}\left(T_{A}\right)>1$ and $4 t \kappa \phi^{\prime \prime}\left(T_{B}\right)>1$, which we assume to be satisfied. We also impose 'stability' conditions (Dixit 1986), which amount to $\left(6 t \kappa \phi^{\prime \prime}\left(T_{B}\right) \kappa-1\right)\left(6 t \mu \phi^{\prime \prime}\left(T_{A}\right)-1\right)-1>0$. Later on, these conditions will be used to sign comparative statics results.
} 


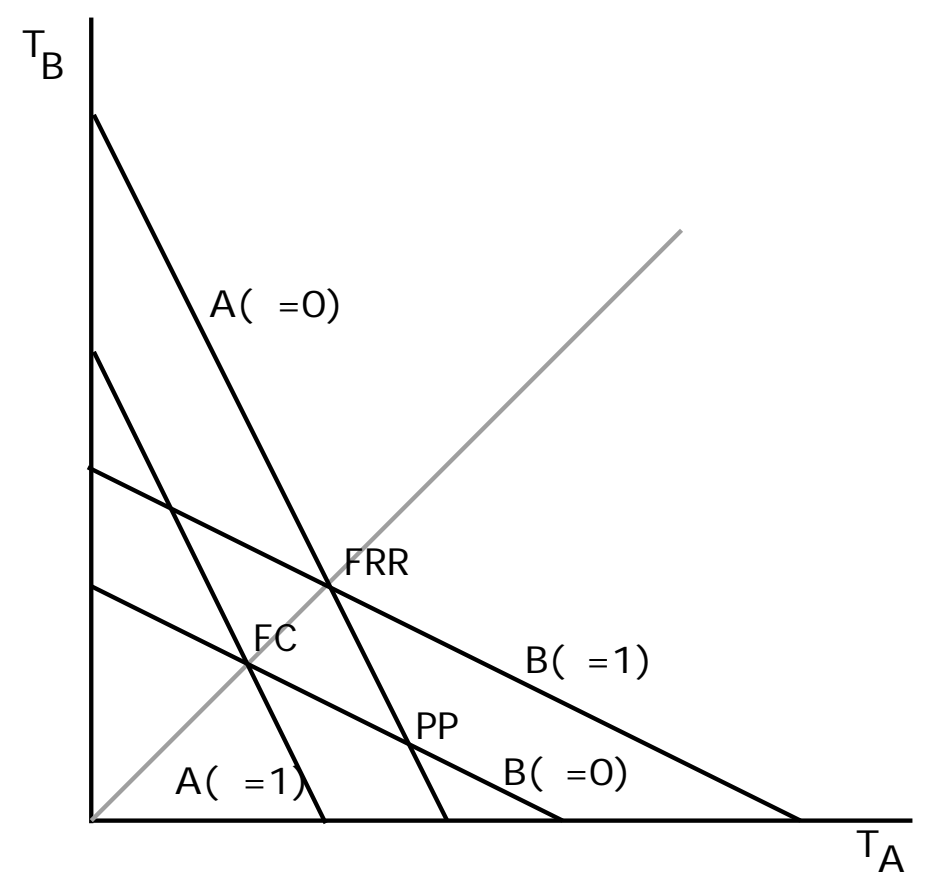

Figure 1: Equilibrium choice of quality.

is $\phi^{\prime}\left(T^{*}\right)=1 /(2 c)$. Naturally, at this symmetric equilibrium, demand is shared equally by both providers. It remains the case of pure preferred provider $(\alpha=0, \beta=0)$, which leads to an asymmetric equilibrium. In this equilibrium, quality of provider $A$ exceeds that of provider $B$, and $P_{A}>P_{B} \cdot{ }^{9}$ The preferred provider achieves a higher market share.

This equilibrium exhibits comparative statics properties that differ from those of the "preferred provider" contract. In this case, $\partial T_{i} / \partial c<0$. That is, an increase in the co-payment rate, meaning that individuals pay more at the point of consumption of health care, implies lower quality of both providers. As price competition becomes stiffer, providers use less the quality level as a competitive instrument for attracting consumers, relying more on the price instrument.

The difference in the comparative statics is due to the way selection of provider $B$ is treated. In the preferred provider contract, the consumer going to provider $B$ receives an amount independent of provider $B$ 's price, while this is not true under the fixed co-payment rate.

Intuitively, the results stem from the following. When the same co-insurance rate is given, no matter the provider selected by the patient, price competition is relaxed. Both providers will set high prices. In addition, quality becomes the main way to attract patients. Therefore, providers will invest in it heavily. The fixed co-payment contract has some interesting properties, as well. This

\footnotetext{
${ }^{9}$ We also impose $\phi^{\prime}\left(T_{A}\right)<1$ to ensure that $P_{B}>(1-c) P_{A}$.
} 
type of scheme does not change, at the margin, the incentive of provider $B$ (the excluded provider) to price aggressively. Charging a higher price implies the same demand response as in the absence of reimbursement. Marginal effects to provider $B$ 's price changes are unchanged. The important difference lies in provider $A$ 's incentives. Increasing its price now has two effects. There is a direct change in demand, as it becomes a more costly provider. But a second (indirect) effect comes into play: the share of the price paid by the patient when he chooses provider $B$ decreases, making this last provider relatively more attractive. This further reduces the demand to provider $A$. It is in the interest of provider $A$ to have a more aggressive pricing behavior. Thus, the symmetric equilibrium under this rule entails lower prices and lower equilibrium qualities. Finally, since in the case of no reimbursement if provider $B$ is selected, it is clear that there is a competitive asymmetry created between the two firms, with an artificial advantage given to the preferred provider. Hence, naturally, provider $A$ will have a higher pair quality-price than will provider $B$.

\subsection{Sequential decisions}

It is now time to look at the case of sequential decision making. Providers choose quality levels first, which are observed by everyone, and then set prices. The game is solved by backward induction as usual. Second-stage prices are equal to those of (7).

First-order conditions for the choice of qualities are:

$$
\begin{aligned}
& \frac{3 t+T_{A}-T_{B}}{9 t \mu}=\phi^{\prime}\left(T_{A}\right), \\
& \frac{3 t+T_{B}-T_{A}}{9 t \kappa}=\phi^{\prime}\left(T_{B}\right) .
\end{aligned}
$$

These conditions imply the same qualitative features of the equilibrium as in the case of simultaneous choices, and we will not repeat them here. The more substantial point is that qualities are lower in this case. Sequential decision making leads to lower equilibrium levels of quality than do simultaneous decisions on price and qualities.

Consider that a fixed co-payment scheme is imposed. From substitution of $(\alpha=0, \beta=1)$ and symmetry of first order conditions, at the equilibrium $T_{A}=T_{B}=\hat{T}$ with $\hat{T}$ defined implicitly by $\phi^{\prime}(\hat{T})=1 /(3 c)$. At this symmetric equilibrium demand is shared equally between both providers. Similarly, for ( $\alpha=1, \beta=0)$, a symmetric equilibrium arises, with quality choice given by $\phi(\tilde{T})=$ $1 / 3$. As to the remaining case, $(\alpha=0, \beta=0)$, the advantage given to the preferred provider again induces an asymmetric equilibrium, where provider $A$ has higher quality. Equilibrium prices follow 
the same pattern as the case of simultaneous decisions. ${ }^{10}$

\subsection{Properties of equilibria}

One of the most controversial issues in financial arrangements for provision of health care is the effect on quality. In this respect, the model provides the results summarized in Table 3.

Table 3: Equilibria properties

\begin{tabular}{|l|c|c|}
\hline & Provider $A$ & Provider $B$ \\
\hline Prices & $P_{A}^{P P}>P_{A}^{F R R}>P_{A}^{F C}$ & $P_{B}^{F R R}>P_{B}^{F C}>P_{B}^{P P}$ \\
Qualities & $T_{A}^{P P}>T_{A}^{F R R}>T_{A}^{F C}$ & $T_{B}^{F R R}>T_{B}^{F C}>T_{B}^{P P}$ \\
\hline
\end{tabular}

Note: the rankings hold for both simultaneous and sequential games.

In terms of qualities, the simultaneous (or sequential timing) does have an effect on the comparison with the social optimum. The same ordering of schemes occurs in both timings. The main difference between the two timings lies in the fact that quality choices are further distorted by the strategic incentive introduced by the sequential move. Equilibrium qualities are lower, holding the reimbursement system constant, in the sequential game relative to the simultaneous one. Under simultaneous decisions, it is easy to check that $T^{W}=T^{F C}$. That is, the social optimum choice is achieved by a fixed co-payment rule. The other alternatives lead to excessive quality (relative to the social optimum) of provider $A$. Provider $B$ will have too high or too low quality, according to whether a fixed reimbursement rate or a pure preferred provider system is adopted, respectively. Table 4 summarizes.

Table 4: Comparison with the socially optimal quality levels

\begin{tabular}{|ll|ccc|}
\hline Simultaneous game & Provider $A$ & + & $=$ & + \\
& Provider $B$ & + & $=$ & - \\
\hline Sequential game & Provider $A$ & + & - & + \\
& Provider $B$ & + & $?$ & $?$ \\
\hline
\end{tabular}

An important result to collect is that the optimal level of quality is achieved by a system that reimburses the amount defined by the preferred provider to any provider selected by the patient. Here,

\footnotetext{
${ }^{10}$ It is now required $2 / 3>\phi^{\prime}\left(T_{A}\right)$ to ensure $P_{B}>(1-c) P_{A}$, at the equilibrium.
} 
the preferred provider fulfills the role of a reference to definition of out-of-plan indemnity rules by the third-payer.

It is important to recognize why the fixed co-payment system performs better. The existence of insurance makes demand less responsive to price and allows for higher prices and competition on quality, rendering too much quality from a social point of view. Trying to correct these incentives by setting a preferred provider, with no reimbursement if the excluded provider is chosen, creates pressure for price competition. This is especially true for the excluded provider. However, as a side effect, it gives an important competitive advantage to the preferred provider. Reimbursing the patient by a fixed amount, regardless of the provider he seeks, with the amount defined by the preferred provider's price, mitigates this artificial advantage. At the same time, it introduces incentives for tougher price competition. This is so because a higher price of the preferred provider also increases the amount received by the patient if he seeks the outside provider. This has a negative effect on the demand of the preferred provider. It creates a dis-incentive for a higher price of the preferred provider.

One implication of the model is that, under some possible type of contracts, quality would be asymmetric across providers. In particular, the preferred provider would exhibit higher quality than would the pure private provider. This equilibrium feature is usually deemed to be an unfair and undesirable. ${ }^{11}$ While it is not difficult to agree that for small problems people can (and do) make a trade-off between quality, cost and horizontal differentiation effects (such as travelling time), for more severe problems the main worry is quality of care, and therefore one may think that our argument (and model) does not apply.

This objection is true, up to a point - people with more severe illnesses will lean towards the higher quality provider. Nonetheless, the main features of the model are still valid, as we may extend the model to introduce marginal value of quality as increasing in illness severity. ${ }^{12}$ Moreover, the existence of two qualities remains as an equilibrium for some types of contract.

As to the sequential decisions timing, Table 4 presents the comparison of quality to the social optimum. We have in mind a small $c$ in order to have a relevant role for insurance in protecting consumers from risk, meaning that $c<2 / 3$ is the more likely situation. ${ }^{13}$ Except for the fixed copayment scheme in the sequential game, quality of provider $A$ is never below the social optimal one (as long as $c<2 / 3$ ). The other robust feature is that a fixed reimbursement rate induces too much quality on the part of both providers, whatever the timing.

\footnotetext{
${ }^{11}$ See, in a different context, the discussion between Selden (1997) and Blomqvist and Johansson (1997a, 1997b).

${ }^{12} \mathrm{~A}$ full proof of this claim is available from the authors at http://ppbarros.fe.unl.pt/papers.html.

${ }^{13}$ The values of $c$ are typically around $20 \%$ to $30 \%$, well below the threshold. For $c>2 / 3$, we have underprovision of quality in all reimbursement systems, except for provider $A$ in PP, where the comparison is ambiguous.
} 
Summarizing, in terms of short versus long-run decisions, we can see that enforcing a fixed copayment system allows the first best solution in the primary health care sector (simultaneous decisions) to be reached. Also, there is no way to attain such an outcome in the specialized care sector (sequential decisions) without regulation. We take this issue up in the next section.

We cannot say anything about prices in comparison with the social optimum. As they are a transfer from one set of agents to another, both carrying equal weight in the social welfare function, such transfers have no social value. If a somewhat higher social valuation is given to a dollar in the pocket of consumers, then prices will be too high from a social point of view, although no distortion to quality choices arises.

Our model also contributes to the discussion of moral hazard effects in health care markets. ${ }^{14}$ Moral hazard is usually seen as providing too much quantity due to the insurance protection at the moment of consumption. There is typically, no focus on the choice of provider. In fact, the choice of provider is the result of a key tension in our society, as we strive to balance the freedom to choose against the need to hold down costs. Thus, there is a (potential) moral hazard of provider choice, which can be addressed in the model.

Under the fixed reimbursement rate contract, we have (apparently) more insurance. Nevertheless, this contract means lower price sensitivity on the part of consumers. In turn, providers are able to charge higher prices and use their quality level to attract patients. Moral hazard, given fixed total demand, appears through higher prices and higher (than socially optimal) quality. Moreover, comparing the fixed reimbursement rate system with the fixed co-payment system, we find that, in equilibrium, the patient pays more (has less insurance) in the former than in the latter. Since equilibrium prices are different, risk borne by patients is also different and it is greater in the fixed reimbursement rate contract.

Besides this moral hazard aspect, we have another one. Under the preferred provider contract, choices of patients are distorted relative to the first best. Thus, there is a (non-monetary) cost borne by patients. Offering zero reimbursement for out-of-plan care may excessively harm the freedom to choose (showing up as welfare loss for those patients closest to the alternative provider). This adds to the moral hazard problem in quality choice.

\footnotetext{
${ }^{14}$ We thank a referee for drawing our attention to this point.
} 


\section{Public Leadership.}

In this section, we intend to deal explicitly with the the mixed public/private provision of health care. We have seen so far that without the intervention of a public agency, competition between two profit maximizing providers (regardless of being public or private) very seldomly yields optimal (welfare maximizing) levels of quality in the the market game. Since quality should be regarded as a main goal in health care markets, we next propose to introduce some degree of regulation in the model. Thus, a governmental body (e.g. National Health System) sets the co-payment applied to patients and regulates the quality and/or price of the preferred provider by direct operation in the market (turning it into a public provider).

We feel that a reasonable way to analyze this scenario is to assign the leadership to the public agency in deciding on the value(s) of the strategic variable(s). We can think of two possibilities regarding the regulatory power of the public agency. In one case, the public agency sets the price and quality levels of the public provider (leader) to maximize welfare, and the private provider adjusts its decisions conditional upon the former. In the other case, the public agency decides the (welfare maximizing) level of quality of the public provider. Then, the private provider will select its quality level and finally both providers will compete in prices in a mixed oligopoly fashion (i.e. the public firm choosing a price to maximize welfare, the private one choosing a profit maximizing price).

These two alternative ways of operating in the market illustrate two phenomena. First, we can envisage regulation more as a concern of the public agency with regard to the services it provides at the different levels of provision (and therefore, regardless of the presence or absence of private providers).

Second, the comparison of the results of two alternative ways of regulating the market will shed light on (some of) the consequences of the present health care deregulatory wave in Western European countries. From a situation where the health care market was heavily regulated both in terms of prices and qualities, we are witnessing a move towards encouraging competition between providers in the hope of achieving some cost containment goal. This competition takes different forms, from leaving the management of public hospitals to private firms, to competing for patients through advertisement campaigns (especially through physicians and insurance companies) on the quality of the different services a particular hospital provides. Nevertheless, the public agency commits to the welfare maximizing objective by setting the corresponding level of quality on the public provider and by encouraging competition in prices on the basis of a mixed oligopoly market.

Several papers have addressed the welfare consequences of a mixed oligopoly in a variety of cir- 
cumstances. White (1996) analyzes the consequences of a privatization process in a mixed oligopoly to find that welfare is not changed if subsidies are used before and after privatization, while subsidies damage welfare if used only before privatization. George et al. (1996) study the interaction between a public firm and a private one when the former has a higher marginal cost than the latter. They show that when the public firm acts as a Stackelberg leader, partial privatization may be welfare improving. Delbono et al. (1996) show that a public leader in the quality stage serves the upper segment of a vertically differentiated market. In a similar context, Grilo (1994) proves that when firms have increasing costs with respect to quality an optimal solution can be achieved using the public firm as a market agent. Finally, in a model of horizontal differentiation, Cremer et al. (1991) compute the number of private firms in a mixed oligopoly with one public firm that makes it socially preferable to a pure private oligopoly. General surveys of earlier literature are Nett (1993) and de Fraja and Delbono (1990).

Under either interpretation, the goal of the analysis is to examine whether the mixed oligopoly structure of the market yields socially optimal results. The question we ask is whether owning a provider, together with definition of out-of-plan indemnity rules, gives a sufficient set of instruments to achieve the first best allocation.

\subsection{Stackelberg leadership}

Consider a regulator deciding on the levels of price and quality of the public provider that maximizes the welfare function given by expression (2). Provider $B$, a private one, takes this price-quality vector as given and acts as a Stackelberg follower to determine its profit maximizing price-quality pair. Obtaining the equilibrium configuration is more demanding now. For simplicity, we assume

$$
\phi\left(T_{i}\right)=\theta T_{i}^{2} / 2 .
$$

As standard, we solve the model by backward induction. In the third stage, provider's $B$ decisions (best-response functions) are given by

$$
\begin{aligned}
& \frac{\partial B_{B}}{\partial P_{B}}=\frac{1}{2}+\frac{T_{B}-T_{A}}{2 t}-\frac{\kappa P_{B}-\mu P_{A}}{2 t}-\frac{\kappa}{2 t} P_{B}=0 \\
& \frac{\partial B_{B}}{\partial T_{B}}=\frac{P_{B}}{2 t}-\theta T_{B}=0 .
\end{aligned}
$$


Solving this system we obtain,

$$
\begin{aligned}
T_{B} & =\frac{T_{A}-t-\mu P_{A}}{1-4 \kappa t \theta}, \\
P_{B} & =2 t \theta T_{B} .
\end{aligned}
$$

Provider $A$ 's aim is to choose a price-quality pair to maximize welfare. That is, the program to solve is to maximize the welfare function (2) where $x, T_{B}$ and $P_{B}$ are given by (6), (10) and (11) respectively. Solving for the first order conditions we obtain, ${ }^{15}$

$$
\begin{aligned}
T_{A}^{*} & =\frac{1-2 \kappa+\kappa^{2} t \theta}{\theta \Upsilon}, \\
P_{A}^{*} & =\frac{(\theta t-1)\left(2 \kappa^{2} t \theta+\kappa-1\right)}{\mu \theta \Upsilon},
\end{aligned}
$$

where $\Upsilon \equiv 1-2 \kappa-\kappa^{2}+2 \kappa^{2} t \theta$. In turn, by substitution, we obtain the provider $B$ 's equilibrium values,

$$
T_{B}^{*}=\frac{\kappa(t \theta-1)}{\theta \Upsilon} ; \quad P_{B}^{*}=2 t \theta T_{B}^{*}
$$

These equilibrium values are well-defined if $t \theta>1$. At these equilibrium values, provider $A$ 's market share is given by,

$$
x^{*}=\frac{1-2 \kappa+\kappa^{2} t \theta}{\Upsilon} \geq \frac{1}{2} .
$$

Finally, profits are given by

$$
\begin{aligned}
& B_{A}=\frac{\left(\kappa^{2} t \theta-2 \kappa+1\right)\left(2-\mu-2 t \theta-\kappa^{2} t \theta(4+\mu-4 t \theta)+2 \kappa(t \theta-1+\mu)\right)}{2 \mu \theta \Upsilon^{2}}, \\
& B_{B}=\frac{\kappa^{2}(t \theta-1)^{2}(4 \kappa t \theta-1)}{2 \theta \Upsilon^{2}} .
\end{aligned}
$$

Clearly, private provider's profits are positive if $t \theta>1 / 4 \kappa$. Also, the levels for the public provider decided by the regulator have to ensure that its profits are non-negative, that is $2-\mu-2 t \theta-\kappa^{2} t \theta(4+$ $\mu-4 t \theta)+2 \kappa(t \theta-1+\mu)>0$. It is easy to verify that this condition is satisfied for the preferred provider and fixed co-payment rules. ${ }^{16}$

The comparison of both providers' decisions (for $\kappa<1$ ) yields,

$$
\begin{aligned}
& P_{A}^{*}-P_{B}^{*}>0 \text { if } t \theta>\max \left\{1, \frac{1-\kappa}{2 \kappa(\kappa-\mu)}\right\} \text { for } \kappa \neq \mu, \\
& P_{A}^{*}-P_{B}^{*}<0 \text { for } \kappa=\mu, \\
& T_{A}^{*}-T_{B}^{*} \geq 0 \text { if } t \theta \leq \frac{1}{\kappa} .
\end{aligned}
$$

\footnotetext{
${ }^{15}$ All proofs and second order conditions are reported in the appendix.

${ }^{16}$ For the fixed rate of reimbursement, an extra condition has to be imposed. See the details in the Appendix.
} 
We proceed now to compare the equilibrium qualities (for $\kappa<1$ ) with the socially optimal values:

$$
\begin{aligned}
& T_{A}^{*}-T^{W}>0, \\
& T_{B}^{*}-T^{W} \geq 0 \text { if } t \theta \geq \frac{1+\kappa}{2 \kappa} .
\end{aligned}
$$

Figure 2 shows the relationships among the different quality levels parametrized by $\kappa<1$. The case $\kappa=1$ yields $T_{A}^{*}=T_{B}^{*}=T^{W}, \mu P_{A}^{*}=P_{B}^{*}$, and hence, $x^{*}=1 / 2$. Thus, public leadership and $\kappa=1$ is sufficient to achieve the first best allocation.

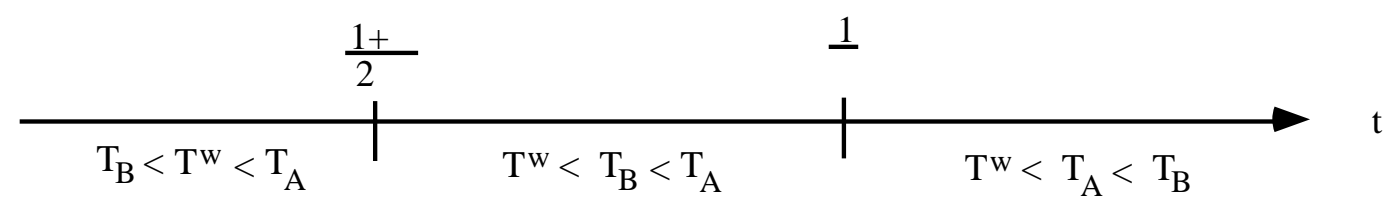

Figure 2: Equilibrium quality levels $(\kappa<1)$.

\section{Remark 1. Summarizing,}

- For $\kappa<1$, the public provider (Stackelberg leader) offers higher quality than the socially optimal one and obtains a higher market share than its rival; the private provider (Stackelberg follower) offers higher quality than the socially optimal one if $t \theta>(1+2 \kappa) / 2 \kappa$.

- For $\kappa=1$, that is, under the fixed co-payment and preferred provider rules, the social optimum choice of qualities is achieved.

We can think about these conclusions turning them around in the following fashion. Assume the social planner sets the optimal quality level at the public provider and chooses the reimbursement rule that would lead the private provider also to choose the right quality, that is, the one attaining an optimal division of patients between the two providers. Then, the planner would choose either the fixed co-payment or the preferred provider rule. Therefore, the first-mover advantage, together with the fact of being a public provider, plus the choice of the reimbursement rules, join to become a sufficient set of instruments for achieving the first best allocation. The public leadership allows the distortion introduced by strategic considerations in the sequential game to be overcome. ${ }^{17}$ It turns out that the fixed reimbursement rule is inferior to any of the other systems in terms of achieving socially optimal qualities.

\footnotetext{
${ }^{17}$ It should be stressed that leadership only or public ownership without leadership do not constitute sufficient instruments.
} 
As the setting of preferred providers or the fixing of co-payment rules both achieve optimal qualities, the selection of the best system needs some further criterion. Using the payments made to providers as an additional criterion, it turns out that a fixed co-payment system does entail lower total payments than does a preferred provider system. Therefore, under this additional criterion the fixed co-payment is again a superior way of setting reimbursement rules.

\subsection{Semi-sequential equilibrium.}

There is another sense in which the public provider can act as a leader. We call it the semi-sequential game. This is a three-stage game where first the regulator selects $T_{A}$, then provider $B$ selects $T_{B}$ and finally, both providers compete in prices. This timing means that provider $A$ acts as a leader in the choice of quality, while price choices are simultaneous. The public provider maximizes social welfare, subject to non-negativity of profits, while the private provider maximizes own profits.

Equilibrium prices in the third stage are given by:

$$
P_{A}=\frac{t-T_{A}+T_{B}}{\mu} ; \quad P_{B}=\frac{t-T_{A}+T_{B}}{\kappa} .
$$

Provider $B$ 's profits in the second stage of the game are

$$
B_{B}\left(T_{B}\right)=P_{B}(1-x)-\frac{\theta}{2} T_{B}^{2} .
$$

The second-stage optimal decision yields

$$
T_{B}=\frac{t-T_{A}}{\kappa \theta t-1} .
$$

Second order conditions require $\kappa \theta t>1$. Going back to the first-stage decision, maximization of social welfare leads to the quality choice of provider $A$ given by

$$
T_{A}^{*}=\frac{2+(2-4 \kappa) t \theta+\kappa^{2} t^{2} \theta^{2}}{\theta \Lambda}, \quad \Lambda=4-4 \kappa t \theta+\kappa^{2} t \theta(2 t \theta-1)>0 .
$$

Substituting this optimal choice for the second-stage optimal decision and feeding both back to the third stage, we obtain the remaining equilibrium values (well-defined since $t \theta>1 / \kappa$ ):

$$
T_{B}^{*}=\frac{2(t \theta-1)(\kappa t \theta-1)}{\theta \Lambda}, \quad P_{A}^{*}=\frac{2 \kappa t(t \theta-1)(\kappa t \theta-1)}{\mu \Lambda}, \quad P_{B}^{*}=\frac{2 t(t \theta-1)(\kappa t \theta-1)}{\Lambda} .
$$

Finally, profits are given by,

$$
\begin{aligned}
B_{A} & =\frac{4 \theta \kappa t(t \theta-1)\left[-4+\kappa+7 \kappa \theta t+\kappa^{3} t^{3} \theta^{3}-\kappa^{2} t \theta(1+4 \theta t)\right]}{2 \mu \theta \Lambda^{2}}- \\
& -\frac{\mu\left(2+2(1-2 \kappa) t \theta+\kappa^{2} t^{2} \theta^{2}\right)^{2}}{2 \mu \theta \Lambda^{2}}, \\
B_{B} & =\frac{2(t \theta-1)^{2}(\kappa t \theta-1)^{3}}{\theta \Lambda^{2}} .
\end{aligned}
$$


Provider $B$ 's profits are positive since $\kappa t \theta>1$. Comparing the providers' decisions we obtain,

$$
P_{A}^{*}-P_{B}^{*} \geq 0 \text { if } \kappa \geq \mu, \quad T_{A}^{*}-T_{B}^{*} \geq 0 \text { if } t \theta \leq 2 / \kappa .
$$

Finally, we compare the equilibrium qualities with the socially optimal ones, to obtain:

$$
T_{A}^{*}-T^{W}>0, \quad T_{B}^{*}-T^{W} \geq 0 \text { if } \theta t \geq \frac{2+\kappa}{2 \kappa} .
$$

In this case, the following is established

Remark 2. It is not possible to attain the social optimal values in the three-stage game. In particular, the public provider always offers a higher quality level than the socially optimal one (in the range of parameters where its profits are non-negative). In turn, the follower provider also offers a higher quality level than the socially optimal one when $\theta t>(2+\kappa) 2 \kappa$.

We can identify some features common to both cases of public leadership. First, the public provider sets an excessive quality from the social point of view. Second, there is a range of parameters under which the excluded provider has a quality level below the social optimum. And, third, there is a range of parameters where quality of the private (excluded) provider exceeds that of the public (preferred) provider. The relative quality level of the private provider is determined by the magnitude of $\theta t$. Intuitively, a high value of $\theta$ means high cost of quality provision, and a high value of $t$ means high costs of horizontal product differentiation. Thus, when quality is more costly and horizontal differentiation relatively more important, the public provider uses it less intensively than does the private provider. This accounts for the reversal of relative positions in quality choices.

Overall, leadership by direct operation of one provider does not ensure achievement of the social optimum, due to the strategic effects resulting from the sequential nature of the decisions. Only under Stackelberg leadership and for a particular set of reimbursement rules are the social optimum qualities achieved. Morover, the fixed co-payment system is again the best one.

\section{Final remarks.}

We have analyzed how a private market outcome for provision of health care is influenced by the insurance arrangements typical of health care financing. In particular, we have looked at the rules defining, or not, preferred sets of providers. In addition, Government action can go beyond setting reimbursement systems to consumers. Ownership of a provider that competes in the marketplace with pure private providers, is one such possibility. 
We identify providers making simultaneous decisions on prices and qualities as an approach to the primary care sector, while sequential decisions (first qualities then prices) approaches the specialized health care sector. Our main conclusion is that enforcing the fixed co-payment rule on the primary health care sector is enough to make providers choose the optimal (welfare maximizing) prices and qualities levels. In contrast, in the specialized health care sector we need to consider a regulated (public) provider to reach the first-best solution in prices and qualities and implement either the fixed co-payment or the fixed reimbursement rules.

We now turn to the implications of our model for health system organization. All governments in European Union Member states have looked at ways to contain health expenditures. Direct and indirect controls over health care providers have been imposed in some countries where co-payments play an important role. In several countries we find controls on prices (pharmaceuticals, per-day treatment in hospitals), while in others no such controls exist. Co-payment changes have been frequent in European countries, mostly limited to the value of the co-payment while maintaining its structure (fixed reimbursement rates). ${ }^{18}$

Moreover, co-payments are designed with insurance coverage in mind (typically, they have an upper limit). No role as a market mechanism underlies the choice of the structure and the value of co-payments. Thus, according to our model, the relative unsuccessful episodes of cost containment through co-payments is not totally surprising. The structure of the co-payment has been kept constant, while our results highlight the fact that changing its structure would have a greater impact.

The market most closely related to our setting is the pharmaceutical market. Reference prices, present in several countries, are much in the spirit of our approach. ${ }^{19}$ Under a reference price system, a single price is set by the insurer (government or other institution) for a group of similar products. Any excess above the reference price has to be paid by the patient. Companies have freedom to set their prices in those countries which have adopted reference price systems. One objective behind the adoption of a reference price system has been to foster competition in the market. Several countries use this system (New Zealand, Germany, The Netherlands, Denmark, Sweden and Italy). Providers (pharmaceutical companies) have argued against the reference price system on the basis that it distorts clinical decision making and limits freedom of choice. Our analysis shows that in this respect a fixed co-payment system performs as well as a fixed reimbursement rate system, and adds the advantage of tougher price competition among providers. It also reveals that exclusion of some providers from

\footnotetext{
${ }^{18}$ See Mossialos and Le Grand (1999) for an overview of recent experiments in cost containment in European countries.

${ }^{19}$ See Bloor et al. (1998) for a short review of reference prices and Mosssialos and Le Grand (1999) for a more detailed discussion.
} 
the reimbursement system (the pure preferred provider case) does induce distortions in the decision to visit a provider, which can be seen as limitations on the freedom of choice.

Although the pharmaceutical market is a very good application of our analysis, we do believe it can be applied in a fruitful way to other providers. For example, visits to general practitioners in some countries (e.g. Ireland, France, Portugal, Sweden) are associated with co-payments, aimed at demand control. As long as GPs retain some control over the prices they charge, namely in private practice, we suggest that fixed reimbursement rate regimes should be changed to fixed co-payment systems.

Our analysis underscores the role of rules defining preferred providers in the way health care markets operate. We believe that our model is a useful framework in which to address market interactions in health care markets where both private and public providers are present. 


\section{Appendix}

\section{Simultaneous decisions}

We claim that

$$
\frac{\partial T_{A}}{\partial \alpha}<0 ; \quad \frac{\partial T_{B}}{\partial \alpha}>0 ; \quad \frac{\partial T_{A}}{\partial \beta}<0 ; \quad \frac{\partial T_{B}}{\partial \beta}>0
$$

The equilibrium choices of qualities, after solving for prices and substituting them in the remaining two first order conditions, are determined by

$$
\begin{aligned}
\nu^{B} & =3 t+T_{B}-T_{A}-6 t \phi^{\prime}\left(T_{B}\right) \kappa=0 \\
\nu^{A} & =3 t+T_{A}-T_{B}-6 t \phi^{\prime}\left(T_{A}\right) \mu=0
\end{aligned}
$$

To sign comparative statics results, we first impose 'stability' requirements to this system (as described in Dixit 1986).

This amounts to thinking of an adjustment process where each firm increases its quality at a given pair $\left(T_{A}, T_{B}\right)$ if $\nu^{i}>0:{ }^{20}$

$$
\dot{T}_{i}=\gamma_{i} \nu^{i}\left(T_{A}, T_{B}\right), \quad i=A, B
$$

where $\gamma_{i}$ is the adjustment speed. Linearizing around the equilibrium $\left(T_{A}^{*}, T_{B}^{*}\right)$ with a first order approximation:

$$
\left[\begin{array}{c}
\dot{T}_{A} \\
\dot{T}_{B}
\end{array}\right]=\left[\begin{array}{ll}
\gamma_{A} \frac{\partial \nu^{A}}{\partial T_{A}} & \gamma_{A} \frac{\partial \nu^{A}}{\partial T_{B}} \\
\gamma_{B} \frac{\partial \nu^{B}}{\partial T_{A}} & \gamma_{B} \frac{\partial \nu^{B}}{\partial T_{B}}
\end{array}\right]\left[\begin{array}{c}
T_{A}-T_{A}^{*} \\
T_{B}-T_{B}^{*}
\end{array}\right]
$$

For stability requirements to be independent of adjustment speeds, we need:

$$
\begin{aligned}
\frac{\partial \nu^{A}}{\partial T_{A}}<0, \quad \frac{\partial \nu^{B}}{\partial T_{B}} & <0 \\
\frac{\partial \nu^{A}}{\partial T_{A}} \frac{\partial \nu^{B}}{\partial T_{B}}-\frac{\partial \nu^{A}}{\partial T_{B}} \frac{\partial \nu^{B}}{\partial T_{A}} & >0
\end{aligned}
$$

The second order conditions imply

$$
\frac{\partial \nu^{A}}{\partial T_{A}}<0, \quad \frac{\partial \nu^{B}}{\partial T_{B}}<0
$$

Therefore, the extra condition we impose is:

$$
\left(1-6 t \mu \phi^{\prime \prime}\left(T_{A}\right)\right)\left(1-6 t \kappa \phi^{\prime \prime}\left(T_{B}\right)\right)-1>0
$$

\footnotetext{
${ }^{20}$ For a similar analysis, although in a different context, see Henriques (1990).
} 
We are now in a position to investigate some comparative statics results. Total differentiation of the reduced-form reaction functions $\nu^{A}$ and $\nu^{B}$ yields:

$$
\begin{aligned}
& d T_{B}-d T_{A}=6 t \phi^{\prime \prime}\left(T_{B}\right) \kappa d T_{B}+6 t \phi^{\prime}\left(T_{B}\right) d \kappa \\
& d T_{A}-d T_{B}=6 t \phi^{\prime \prime}\left(T_{A}\right) \mu d T_{A}+6 t \phi^{\prime}\left(T_{A}\right) d \mu
\end{aligned}
$$

Rewriting:

$$
\left[\begin{array}{cc}
1 & 6 t \kappa \phi^{\prime \prime}\left(T_{B}\right)-1 \\
6 t \phi^{\prime \prime}\left(T_{A}\right) \mu-1 & 1
\end{array}\right]\left[\begin{array}{l}
d T_{B} \\
d T_{A}
\end{array}\right]=\left[\begin{array}{l}
-6 t \phi^{\prime}\left(T_{B}\right) d \kappa \\
-6 t \phi^{\prime}\left(T_{A}\right) d \mu
\end{array}\right]
$$

Solving for the changes in the two endogenous variables, we get:

$$
\begin{aligned}
d T_{A} & =\frac{6 t \phi^{\prime}\left(T_{A}\right)\left(6 t \phi^{\prime \prime}\left(T_{B}\right) \kappa-1\right) d \mu+6 t \phi^{\prime}\left(T_{B}\right) d \kappa}{\left(6 t \phi^{\prime \prime}\left(T_{B}\right)-1\right)\left(6 t \phi^{\prime \prime}\left(T_{A}\right) \mu-1\right)-1} \\
d T_{B} & =\frac{6 t \phi^{\prime}\left(T_{A}\right) d \mu+\left(1-6 t \mu \phi^{\prime \prime}\left(T_{A}\right)\right) 6 t \phi^{\prime}\left(T_{B}\right) d \kappa}{\left(6 t \phi^{\prime \prime}\left(T_{B}\right)-1\right)\left(6 t \phi^{\prime \prime}\left(T_{A}\right) \mu-1\right)-1}
\end{aligned}
$$

From the above condition, it is straightforward to see that

$$
\frac{d T_{A}}{d \mu}<0 \quad \frac{d T_{B}}{d \mu}>0 \quad \frac{d T_{A}}{d \kappa}>0 \quad \frac{d T_{B}}{d \kappa}<0
$$

It also follows that

$$
\frac{d T_{A}}{d \alpha}<0 ; \quad \frac{d T_{b}}{d \alpha}>0 ; \quad \frac{\partial T_{A}}{\partial \beta}<0 ; \quad \frac{\partial T_{B}}{\partial \beta}>0
$$

\section{Sequential decisions}

In this case,

$$
\begin{aligned}
& \nu^{A}=3 t+T_{A}-T_{B}-9 t \mu \phi^{\prime}\left(T_{A}\right) \\
& \nu^{B}=3 t+T_{B}-T_{A}-9 t \kappa \phi^{\prime}\left(T_{B}\right)
\end{aligned}
$$

The associated stability condition is:

$$
\left(1-9 t \mu \phi^{\prime \prime}\left(T_{A}\right)\right)\left(1-9 t \kappa \phi^{\prime \prime}\left(T_{B}\right)\right)-1>0
$$

It is easy to see that the same comparative statics results emerge. 


\section{Stackelberg Leadership}

By substituting (10) (11) and (16) into the welfare function (37) we derive the first order conditions of provider $A$ 's problem:

$$
\begin{aligned}
\frac{\partial W\left(P_{A}, T_{A}\right)}{\partial P_{A}} & =\frac{\mu \theta\left[\mu P_{A}\left(2 \kappa-2 \kappa^{2} t \theta-1\right)+T_{A}\left(1-\kappa-2 \kappa^{2} t \theta\right)+t\left(\kappa+2 \kappa^{2} t \theta-1\right)\right]}{(4 \kappa t \theta-1)^{2}} \\
\frac{\partial W\left(P_{A}, T_{A}\right)}{\partial T_{A}} & =\frac{1+\mu \theta P_{A}\left(1-\kappa-2 \kappa^{2} t \theta\right)+2 \theta T_{A}\left(4 \kappa t \theta+3 \kappa^{2} t \theta-8 \kappa^{2} t^{2} \theta^{2}-1\right)}{(4 \kappa t \theta-1)^{2}}+ \\
& +\frac{t \theta\left(1-8 \kappa+10 \kappa^{2} t \theta\right)}{(4 \kappa t \theta-1)^{2}}
\end{aligned}
$$

The hessian matrix of second derivatives is given by,

$$
H=\left(\begin{array}{cc}
\frac{\partial^{2} W}{\partial P_{A}^{2}} & \frac{\partial^{2} W}{\partial P_{A} \partial T_{A}} \\
\frac{\partial^{2} W}{\partial T_{A} \partial P_{A}} & \frac{\partial^{2} W}{\partial T_{A}^{2}}
\end{array}\right)=\left(\begin{array}{cc}
\frac{\mu^{2} \theta\left(2 \kappa-2 \kappa t \theta^{2}-1\right)}{(4 \kappa t \theta-1)^{2}} & \frac{\mu \theta\left(1-\kappa-2^{2} t \theta\right)}{(4 \kappa t \theta-1)^{2}} \\
\frac{\mu \theta\left(1-\kappa-2 \kappa^{2} t \theta\right)}{(4 \kappa t \theta-1)^{2}} & \frac{2 \mu\left(4 \kappa t \theta+3 \kappa^{2} t \theta-8 \kappa^{2} t^{2} \theta^{2}-1\right)}{(4 \kappa t \theta-1)^{2}}
\end{array}\right)
$$

so that second order conditions are,

$$
\begin{aligned}
& 2 \kappa-2 \kappa^{2} t \theta-1<0 \\
& \kappa t \theta(4+3 \kappa-8 \kappa t \theta)-1<0 \\
& 1-2 \kappa-\kappa^{2}+2 \kappa^{2} t \theta \equiv \Upsilon>0
\end{aligned}
$$

Solving the system of first order conditions, we obtain providers' equilibrium values. Given that from (13) $\Upsilon>0, P_{B}^{*}$ and $T_{B}^{*}$ are well-defined if

$$
t \theta>1
$$

In turn, (14) implies $1-2 \kappa+\kappa^{2} t \theta>(1-\kappa)^{2}$, so that $T_{A}^{*}$ is also well-defined. Finally, given (14) and (12), $P_{A}^{*}$ is also positive.

The comparison of both providers' decisions yields,

$$
\begin{aligned}
& P_{A}^{*}-P_{B}^{*}=\frac{(t \theta-1)\left(\kappa-2 \mu t \theta \kappa+2 t \theta \kappa^{2}-1\right)}{\mu \theta \Upsilon} \\
& T_{A}^{*}-T_{B}^{*}=\frac{(\kappa-1)(t \theta \kappa-1)}{\theta \Upsilon} \geq 0 \text { if } t \theta \leq \frac{1}{\kappa} .
\end{aligned}
$$

Given (14) and that $\Upsilon>0$, the sign of $P_{A}^{*}-P_{B}^{*}$ is determined by the second term in the numerator which can be rewritten as $\kappa-1+2 \kappa t \theta(\kappa-\mu)$. From the definitions of $\mu$ and $\kappa$ it follows that,

$$
\begin{aligned}
& \text { for } \kappa>\mu, P_{A}^{*}>P_{B}^{*} \text { if } t \theta>\max \left\{1, \frac{1-\kappa}{2 \kappa(\kappa-\mu)}\right\}, \\
& \text { for } \kappa<\mu, P_{A}^{*}>P_{B}^{*} \\
& \text { for } \kappa=\mu, P_{A}^{*} \leq P_{B}^{*} .
\end{aligned}
$$


We proceed now to compare the equilibrium qualities with the socially optimal values given by $T^{W}=$ $1 / 2 \theta$.

$$
\begin{aligned}
T_{A}^{*}-T^{W} & =\frac{(1-\kappa)^{2}}{2 \theta \Upsilon} \geq 0 \\
T_{B}^{*}-T^{W} & =\frac{(\kappa-1)(1+\kappa-2 \kappa t \theta)}{2 \theta \Upsilon} \geq 0 \text { if } t \theta>\frac{1+\kappa}{2 \kappa} .
\end{aligned}
$$

\section{Semi-sequential equilibrium.}

We first examine second order conditions in each stage. In the third stage, the second order conditions are:

$$
\frac{\partial^{2} W}{\partial P_{A}^{2}}=-\mu^{2} / 2 t<0 \quad \frac{\partial^{2} B_{B}}{\partial P_{B}^{2}}=-\kappa / t<0
$$

The stability requirement of Dixit (1986) amounts to

$$
\frac{\mu \kappa}{2 t^{2}}\left(1-\frac{\mu}{2}\right)>0
$$

which is always satisfied.

In the second stage, the second order condition is

$$
\frac{\partial^{2} B_{B}}{\partial T_{B}^{2}}=\frac{1}{\kappa t}-\theta<0, \text { or } \theta \kappa t>1
$$

Finally, in the first stage, we have

$$
\frac{\partial^{2} W}{\partial T_{A}^{2}}=-\frac{\theta \Lambda}{2(\kappa t \theta-1)^{2}}
$$

which is fulfilled if $\Lambda>0$ where $\Lambda=4-4 \kappa t \theta-\kappa^{2} t \theta(1-2 t \theta)$. The roots of $\Lambda$, seen as polynomial in $\theta t$, are:

$$
\begin{aligned}
& (\theta t)^{a}=\left(4+\kappa-\sqrt{\kappa^{2}+8 \kappa-16}\right) / 4 \kappa \\
& (\theta t)^{b}=\left(4+\kappa+\sqrt{\kappa^{2}+8 \kappa-16}\right) / 4 \kappa
\end{aligned}
$$

The roots of $\kappa^{2}+8 \kappa-16$ are $\kappa=-9.54$ and $\kappa=1.65$, implying negative values for the relevant range of $\kappa$. Thus, $\Lambda$ has no real roots for $\kappa \in[c, 1]$, and $\Lambda>0$ for the entire relevant range.

We now check positivity of equilibrium values.

$$
T_{A}^{*}=\frac{2+(2-4 \kappa) t \theta+\kappa^{2} t^{2} \theta^{2}}{\theta \Lambda}
$$


Since $\Lambda>0$, it is sufficient to check the numerator. The roots of the numerator in $t \theta$ are

$$
\begin{aligned}
y^{a} & =\frac{-1+2 \kappa-\sqrt{1-4 \kappa+2 \kappa^{2}}}{\kappa^{2}} \\
y^{b} & =\frac{-1+2 \kappa+\sqrt{1-4 \kappa+2 \kappa^{2}}}{\kappa^{2}}
\end{aligned}
$$

The roots $y^{a}$ and $y^{b}$ are real values for $\kappa \leq 0.292$. Thus, for $\kappa>0.292, T_{A}^{*}$ is always positive. For $\kappa \leq 0.292$, it is also straightforward to check that $\max \left\{y^{a}, y^{b}\right\}<0$. Thus, in the relevant range of $\theta t>0$, the equilibrium value of $T_{A}^{*}$ is always positive.

Direct inspection of equilibrium values of $T_{B}^{*}, P_{A}^{*}, P_{B}^{*}$ reveals that parameter restrictions imposed to satisfy second order conditions are sufficient for positivity of equilibrium values.

The comparison $P_{A}^{*}-P_{B}^{*}$ and $T_{A}^{*}-T_{B}^{*}$ give no definite conclusion:

$$
\begin{aligned}
& P_{A}^{*}-P_{B}^{*}=\frac{2 t(\kappa-\mu)(t \theta-1)(\kappa t \theta-1)}{\mu \Lambda}>0 \text { if } \kappa>\mu \\
& T_{A}^{*}-T_{B}^{*}=\frac{t(k-2)(k t \theta-2)}{\Lambda}>0 \text { if } t \theta<2 / \kappa
\end{aligned}
$$

Finally, we compare the equilibrium qualities with the socially optimal ones, to obtain:

$$
\begin{aligned}
T_{A}^{*}-T^{W} & =\frac{(\kappa-2)^{2} t}{2 \Lambda}>0 \\
T_{B}^{*}-T^{W} & =\frac{t(2-\kappa)(\kappa+2-2 \kappa t \theta)}{2 \Lambda}
\end{aligned}
$$

Thus, $T_{B}^{*}>T^{W}$ if $\kappa+2-2 \kappa t \theta<0$, or

$$
\theta t>\frac{2+\kappa}{2 \kappa}
$$

Of course, if $T_{B}^{*}>T_{A}^{*}$ it must be the case that $T_{B}^{*}>T^{W}$. 


\section{References}

Barros, P.P. and X. Martinez-Giralt, (2000), Selecting Negotiation Processes, mimeo.

Blomqvist, A. and P-O. Johansson (1997a), Economic Efficiency and Mixed Public/Private Insurance, Journal of Public Economics, 66: 505-516.

Blomqvist, A. and P-O. Johansson (1997b), Rejoinder to T.M. Selden, Journal of Public Economics, 66: $525-526$.

Bloor, K., A. Maynard and N. Freemantle, (1996), Lessons from International Experience in Controlling Pharmaceutical Expenditure III: Regulating Industry, Bristish Medical Journal, 313: 33-35 (July 6).

Che, Y.-K. and I. Gale (1997) Buyer Alliances and Managed Competition, Journal of Economics \& Management Strategy, 6(1): 175-200.

Cremer, H., M. Marchand and J.F. Thisse (1991), Mixed Oligopoly with Differentiated Products, International Journal of Industrial Organization, 9(1), 43-53.

de Fraja, G. and F. Delbono (1990), Game Theoretic Models of Mixed Oligopoly, Journal of Economic Surveys, 4(1), 1-17.

Delbono, F., V. Denicolo and C. Scarpa (1996), Quality Choice in a Vertically Differentiated Mixed Oligopoly, Economic Notes, 25(1), 33-46.

Dixit, A. (1986), Comparative Statics for Oligopoly, International Economic Review, 27(1): 107-122.

George, K., C. La Manna and M.A. Manfredi (1996), Inefficiency and Public Ownership, Review of Industrial Organization, 11(6), 853-860.

Glazer, J. and T.G. McGuire, (1993), Should Physicians Be Permitted to 'Balance Bill' Patients?, Journal of Health Economics, 11, 239-258.

Grilo, I. (1994), Mixed Duopoly under Vertical Differentiation, Annales d'Economie et de Statistique, 33, 91-112.

Henriques, I. (1990), Cooperative and Noncooperative R\&D in Duopoly with Spillovers: Comment, American Economic Review, 80(3): 638-640.

Isaacs, S.L., (1996), Consumers' Information Needs: Results of a National Survey, Health Affairs, 15: $31-41$

Ma, A. and T. McGuire (1997), Introduction to the Special Issue: The Industrial Organization of Health Care II, Journal of Economics \& Management Strategy, 6(1): 1-5.

Ma, A. and J.F. Burgess (1993), Quality Competition, Welfare, and Regulation, Journal of Economics, 58: $153-173$.

Mossialos, E. and Le Grand, J., (1999), Cost Containment in the EU: an Overview, in Health Care and Cost Containment in the European Union, edited by E. Mossialos and J. Le Grand, Ashgate.

Nett, L. (1993), Mixed Oligopoly with Homogeneous Goods, Annals of Public and Cooperative Economics, 64(3), 367-393.

Selden, T.M. (1997), More on the Economic Efficiency of Mixed Public/Private Insurance, Journal of Public Economics, 66: 517-523.

White, M.D. (1996), Mixed Oligopoly, Privatization and Subsidization, Economics Letters, 53(2), 189-195.

Wolinsky, A. (1997), Regulation of Duopoly: Managed Competition vs Regulated Monopolies, Journal of Economics \& Management Strategy, 6(4): 821-847. 